\title{
On quasiconformal maps and semi-linear equations in the plane
}

\author{
Vladimir Gutlyanskĭ̌, Olga Nesmelova, Vladimir Ryazanov
}

\begin{abstract}
Assume that $\Omega$ is a domain in the complex plane $\mathbb{C}$ and $A(z)$ is symmetric $2 \times 2$ matrix function with measurable entries, $\operatorname{det} A=1$ and such that $1 / K|\xi|^{2} \leq\langle A(z) \xi, \xi\rangle \leq K|\xi|^{2}, \xi \in \mathbb{R}^{2}, 1 \leq K<\infty$. In particular, for semi-linear elliptic equations of the form $\operatorname{div}(A(z) \nabla u(z))=$ $f(u(z))$ in $\Omega$ we prove Factorization Theorem that says that every weak solution $u$ to the above equation can be expressed as the composition $u=T \circ \omega$, where $\omega: \Omega \rightarrow G$ stands for a $K$-quasiconformal homeomorphism generated by the matrix function $A(z)$ and $T(w)$ is a weak solution of the semi-linear equation $\triangle T(w)=J(w) f(T(w))$ in $G$. Here the weight $J(w)$ is the Jacobian of the inverse mapping $\omega^{-1}$. Similar results hold for the corresponding nonlinear parabolic and hyperbolic equations. Some applications of these results in anisotropic media are given.
\end{abstract}

2010 MSC. Primary 30C62, 31A05, 31A20, 31A25, 31B25, 35J61, 35Q15; Secondary 30E25, 31C05, 34M50, 35F45.

Key words and phrases. Semi-linear elliptic, parabolic and hyperbolic equations, nonlinear Laplace equation, anisotropic media, quasiconformal mappings.

\section{Introduction}

It is well known that the first order linear partial differential equation

$$
\omega_{\bar{z}}=\mu(z) \omega_{z}, \quad z \in \Omega \subseteq \mathbb{C},
$$

where $\omega_{z}=\frac{1}{2}\left(\omega_{x}-i \omega_{y}\right), \omega_{\bar{z}}=\frac{1}{2}\left(\omega_{x}+i \omega_{y}\right), z=x+i y$, which has come to be known as the Beltrami equation, turned out to be instrumental in the study of Riemann surfaces, Teichmüller spaces, Kleinian groups, meromorphic functions, low dimensional topology, holomorphic motion complex dynamics, Clifford analysis and control theory. As known, a 
$K$-quasiconformal mapping $\omega: \Omega \rightarrow \mathbb{C}, K \geq 1$, is just an orientationpreserving homeomorphic $W_{\text {loc }}^{1,2}(\Omega)$ solution to the Beltrami equation when the measurable coefficient $\mu$ satisfies the strong ellipticity condition $|\mu(z)| \leq(K-1) /(K+1)$ almost everywhere in $\Omega$. Note in particular, that if $\mu=0$ in a domain $\Omega \subset \mathbb{C}$, then the Beltrami equation reduces to the Cauchy-Riemann equation and a solution $\omega$ is analytic in $\Omega$, see e.g. [1,25], see also [19], and the references therein.

We also would like to pay attention to a strong interaction between linear and non-linear elliptic systems in the plane and quasiconformal mappings. The most general first order linear homogeneous elliptic system with real coefficients can be written in the form $\omega_{\bar{z}}+\mu(z) \omega_{z}+\nu(z) \overline{\omega_{z}}=0$, with measurable coefficients $\mu$ and $\nu$ such that $|\mu|+|\nu| \leq(K-1) /(K+$ $1)<1$. This equation is a particular case of a non-linear first order system $\omega_{\bar{z}}=H\left(z, \omega_{z}\right)$ where $H: G \times \mathbb{C} \rightarrow \mathbb{C}$ is Lipschitz in the second variable,

$$
\left|H\left(z, w_{1}\right)-H\left(z, w_{2}\right)\right| \leq \frac{K-1}{K+1}\left|w_{1}-w_{2}\right|, \quad H(z, 0) \equiv 0 .
$$

The principal feature of the above equation is that the difference of two solutions need not solve the same equation but each solution can be represented as a composition of a quasiconformal homeomorphism and an analytic function. Thus quasiconformal mappings become the central tool for the study of these non-linear systems. A rather comprehensive treatment of the present state of the theory is given in the excellent book of Astala, Iwaniec and Martin [2]. This book contains also an exhaustive bibliography on the topic. In particular, the following fundamental Harmonic Factorization Theorem for the uniformly elliptic divergence equations

$$
\operatorname{div} A(z, \nabla u)=0, \quad z \in \Omega,
$$

holds, see [2], Theorem 16.2.1: Every solution $u \in W_{\text {loc }}^{1,2}(\Omega)$ of the equation (1.2) can be represented as $u(z)=h(\omega(z))$, where $\omega: \Omega \rightarrow G$ is $K$-quasiconformal and $h$ is harmonic on $G$.

The main goal of this paper is to point out another application of quasiconformal mappings to the study of some nonlinear partial differential equations in the plane. Namely, we will deal with co-called semi-linear partial differential equations, linear part of which contains the elliptic operator in the divergence form $\operatorname{div}[A(z) \nabla u(z)]$, where the matrix function $A(z)$ is assumed to be symmetric, measurable and satisfy the uniform ellipticity condition

$$
\frac{1}{K}|\xi|^{2} \leq\langle A(z) \xi, \xi\rangle \leq K|\xi|^{2} \text { a.e. in } \Omega
$$


for every $\xi \in \mathbb{C}$, where $1 \leq K<\infty$. Some examples of such semi-linear equations include in anisotropic case the nonlinear heat equation like

$$
u_{t}-\operatorname{div}[A(z) \nabla u(z)]=f(u),
$$

(the same equation describes the brownian motion, diffusion models of population dynamics, and many other phenomena), nonlinear wave equation

$$
u_{t t}-\operatorname{div}[A(z) \nabla u(z)]=f(u),
$$

with continuous functions $f$ or nonlinear Schrödinger equation

$$
i u_{t}+\operatorname{div}[A(z) \nabla u(z)]=k|u|^{p} u,
$$

as well as their stationary counterparts. We show that every weak solution of semi-linear equations of such type can be factorized as the composition of a solution to the corresponding isotropic equation and a quasiconformal mapping agreed with the matrix function $A(z)$. If we deal, for example, with the semi-linear equation

$$
\operatorname{div}[A(z) \nabla u(z)]=f(u), \quad f \geq 0,
$$

then we prove the following generalization of the mentioned above Factorization theorem: Every solution $u \in W_{\mathrm{loc}}^{1,2}(\Omega)$ of the semi-linear equation (1.7) can be represented as $u(z)=h(\omega(z))$, where $\omega: \Omega \rightarrow G$ is a quasiconformal mapping agreed with the matrix function $A$ and $h$ is subharmonic on $G$.

The paper is organized as follows. In the next section we recall basic results from the theory of quasiconformal mappings and their connection with linear elliptic differential equations in the divergence form. In Section 3 we prove the basic identity concerning the linear elliptic operator in the divergence form. The main result of this section is Proposition 3.1. In Section 4 we prove the principal Factorization Theorem 4.1 and their corollaries for some elliptic semi-linear model equations in the plane. Some applications of Factorization Theorem to existence theorems for elliptic semi-linear equations are given in Section 5. Its applications to the free boundary problems ("dead zones") can be found in Section 6. A similar discussion of equations of the heat type is in Section 7. Finally, Section 8 contains a discussion of boundary value problems.

\section{QK-maps and divergent equations}

Let $\Omega$ be a domain in the complex plane $\mathbb{C}$. Denote by $M^{2 \times 2}(\Omega)$ the class of all $2 \times 2$ symmetric matrix function $A(z)=\left\{a_{j k}(z)\right\}$ with 
measurable entries and $\operatorname{det} A(z)=1$, satisfying the uniform ellipticity condition

$$
\frac{1}{K}|\xi|^{2} \leq\langle A(z) \xi, \xi\rangle \leq K|\xi|^{2} \quad \text { a.e. in } \Omega
$$

for every $\xi \in \mathbb{C}$ where $1 \leq K<\infty$.

Given $A \in M^{2 \times 2}(\Omega)$, let us first consider the following second order elliptic homogeneous equation in the divergent form

$$
\operatorname{div}(A(z) \nabla u)=0 \quad \text { a.e. in } \Omega .
$$

Equation (2.2) is interpreted in the distributional sense. That is, a function $u$ is a weak solution to the equation if it has locally integrable gradient $\nabla u$ with

$$
\int_{\Omega}\langle A(z) \nabla u, \nabla \varphi\rangle=0 \quad \forall \varphi \in C_{0}^{\infty}(\Omega) .
$$

This is meaningful at least for $u \in W_{\text {loc }}^{1,1}(\Omega)$, where $W_{\text {loc }}^{1, p}(\Omega)$ stands for the well-known Sobolev space. Here we will assume a little more regularity, namely that $u \in C \cap W_{\text {loc }}^{1,2}(\Omega)$, because in this paper we will be dealing with quasiconformal mappings generated by $A$ with the uniform ellipticity condition (2.1). We plan to study also semi-linear degenerate partial differential equations in subsequent works. In that case we will consider the weaker solutions $u \in C \cap W_{\text {loc }}^{1,1}(\Omega)$.

Let $A \in M^{2 \times 2}(\Omega)$ and $u \in C \cap W_{\text {loc }}^{1,2}(\Omega)$ be a weak solution to (2.2). Then there exists $v \in C \cap W_{\text {loc }}^{1,2}(\Omega)$, called the stream function of $u$, such that

$$
\nabla v=H A \nabla u \quad \text { a.e in } \Omega, \text { where } H=\left(\begin{array}{cc}
0 & -1 \\
1 & 0
\end{array}\right) .
$$

Note by the way that

$$
H^{2}=-I, \text { where } I=\left(\begin{array}{ll}
1 & 0 \\
0 & 1
\end{array}\right),
$$

i.e. $H$ takes a part of the imaginary unit in the space of $2 \times 2$ matrices.

Setting $\omega(z)=u(z)+i v(z)$, one writes in complex notations that $\omega$ satisfies the Beltrami equation

$$
\omega_{\bar{z}}(z)=\mu(z) \omega_{z}(z) \quad \text { a.e. in } \Omega,
$$

where the complex dilatation $\mu(z)$ is given by

$$
\mu(z)=\frac{a_{22}(z)-a_{11}(z)-2 i a_{12}(z)}{\operatorname{det}(I+A(z))} .
$$


The condition of ellipticity (2.1) now is written as

$$
|\mu(z)| \leq \frac{K-1}{K+1} \quad \text { a.e. in } \Omega .
$$

And vice versa, given measurable complex valued function $\mu$, satisfying (2.7), one can invert the algebraic system (2.6) to obtain that, see e.g. Theorem 16.1.6 in [2],

$$
A(z)=\left(\begin{array}{cc}
\frac{|1-\mu|^{2}}{1-|\mu|^{2}} & \frac{-2 \operatorname{Im} \mu}{1-|\mu|^{2}} \\
\frac{-2 \operatorname{Im} \mu}{1-|\mu|^{2}} & \frac{|1+\mu|^{2}}{1-|\mu|^{2}}
\end{array}\right) .
$$

Thus, given any $A \in M^{2 \times 2}(\Omega)$, one produces by (2.6) the complex dilatation $\mu(z)$ for which in turn, by the Measurable Riemann mapping theorem, see e.g. Theorem V.B.3 in [1] and Theorem V.1.3 in [25], the Beltrami equation (2.5) generates as its solution a quasiconfomal homeomorphism $\omega: \Omega \rightarrow G$. As the domain $G$ one can take any plane domain which is conformally equivalent to $\Omega$.

It is well known that $\omega \in W_{\mathrm{loc}}^{1, p}(\Omega), 2 \leq p<\frac{2 K}{K-1}, \omega \in C_{\mathrm{loc}}^{\alpha}(\Omega), \alpha=$ $1 / K$, and we have seen that if $\omega(z)=u(z)+i v(z)$, then $\operatorname{div}(A(z) \nabla u(z))=$ $0, \operatorname{div}(A(z) \nabla v(z))=0$ a.e. in $\Omega$ and $u$ and $v \in C \cap W_{\text {loc }}^{1,2}(\Omega)$. In what follows we will say that the matrix function $A$ generates the corresponding quasiconformal mapping $\omega$, or that $A$ and $\omega$ are agreed.

Note also in passing the very often applied fact that quasiconformal mappings $\omega$ admit the change of variables in integrals because homeomorphisms of the class $W_{\text {loc }}^{1,2}$ are absolute continuous with respect to the area measure, see e.g. Theorem III.6.1, Lemmas III.2.1 and III.3.3 in [25].

We complete this section with the following very important result on the composition operators in the Sobolev spaces.

Let $\Omega$ be a domain in the $n$-dimensional Euclidean space $\mathbb{R}^{n}, n \geq 2$. For the sake of completeness in the exposition we recall that the Sobolev space $L_{p}^{1}(\Omega), p \geq 1$, consists of locally integrable functions $\varphi: \Omega \rightarrow \mathbb{R}$ with distributional partial derivatives of the first order with the seminorm

$$
\|\varphi\|_{L_{p}^{1}(\Omega)}=\|\nabla \varphi\|_{L_{p}(\Omega)}=\left(\int_{\Omega}|\nabla \varphi|^{p} d m\right)^{1 / p}<\infty,
$$

where $m$ is the Lebesgue measure in $\mathbb{R}^{n}, \nabla \varphi$ is the distributional gradient of the function $\varphi, \nabla \varphi=\left(\partial \varphi / \partial x_{1}, \ldots, \partial \varphi / \partial x_{n}\right), x=\left(x_{1}, \ldots, x_{n}\right)$, defined by the conditions

$$
\int_{\Omega} \varphi \cdot \frac{\partial \eta}{\partial x_{i}} d m=-\int_{\Omega} \frac{\partial \varphi}{\partial x_{i}} \cdot \eta d m \quad \forall \eta \in C_{0}^{\infty}(\Omega), i=1,2, \ldots, n .
$$


Here $C_{0}^{\infty}(\Omega)$ stands for the space of all infinitely smooth functions with a compact support in $\Omega$. Similarly, a vector-function is said to belong to the Sobolev class $L_{p}^{1}(\Omega)$ if its coordinate functions belong to $L_{p}^{1}(\Omega)$. The classes $W^{1, p}(\Omega)=L_{p}^{1}(\omega) \cap L_{p}(\Omega)$, which will be used later on, differ from the classes $L_{p}^{1}(\Omega)$ only by the norm

$$
\|\varphi\|_{W^{1, p}(\Omega)}=\|\varphi\|_{L_{p}(\Omega)}+\|\nabla \varphi\|_{L_{p}(\Omega)} .
$$

The following statement will play an important role in our further considerations, see [11,34] and [39].

Lemma 2.1. Let $\omega: \Omega \rightarrow G$ be a homeomorphism. Then the following conditions are equivalent:

1) the composition $\omega^{*} \varphi=\varphi \circ \omega$ generates the bounded operator

$$
\omega^{*}: L_{p}^{1}(G) \rightarrow L_{q}^{1}(\Omega), \quad 1 \leq q \leq p<\infty,
$$

2) the mapping $\omega$ belongs to the class $W_{\mathrm{loc}}^{1,1}(\Omega)$ and the function

$$
K_{p}(x ; \omega):=\inf \left\{k(x):\|D \omega\|(x) \leq k(x)\left|J_{\omega}(x)\right|^{1 / p}\right\}
$$

belongs to $L_{r}(\Omega)$, where $r$ is determined from the equality

$$
\frac{1}{r}=\frac{1}{q}-\frac{1}{p} .
$$

Here $\|D \omega\|(x)$ stands for the operator norm of the Jacobian matrix $D \omega$ of the mapping $\omega$ at the point $x$, i.e., $\|D \omega\|(x):=\sup _{h \in \mathbb{C},|h|=1} D \omega \cdot h$.

Assuming that $\omega$ is a quasiconformal homeomorphism and, consequently, $\omega \in W_{\text {loc }}^{1,2}(\Omega)$ and $K_{2}(x, \omega) \in L^{\infty}(\Omega)$, we arrive at the the following conclusion, when $n=2, p=2, q=2$ and $r=\infty$.

Proposition 2.1. Let $\omega: \Omega \rightarrow G$ be a quasiconformal homeomorphism and let $\varphi: G \rightarrow \mathbb{C}$ belong to the class $W_{\mathrm{loc}}^{1,2}(G)$. Then the composition function $\varphi \circ \omega \in W_{\mathrm{loc}}^{1,2}(G)$.

The study of superposition operators on Sobolev spaces stems from the classical article [33], see also e.g. [10,36] and [37] for the detailed history and bibliography. The Reshetnyak problem on the description of all isomorphisms of the Sobolev space $L_{n}^{1}$ generated by a quasiconformal mapping in $\mathbb{R}^{n}, n \geq 2$, was posed in 1968 at the first Donetsk colloquium in the theory of quasiconformal mappings. The problem was solved in [38], see also [32]. As a consequence, the composition $\omega^{*} \varphi=\varphi \circ \omega$ generates the bounded operator

$$
\omega^{*}: L_{2}^{1}(G) \rightarrow L_{2}^{1}(\Omega)
$$

if and only if the homeomorphism $\omega$ is quasiconformal, see also [31]. 


\section{A basic identity}

It is well-known that every positive defined quadratic form

$$
d s^{2}=a(x, y) d x^{2}+2 b(x, y) d x d y+c(x, y) d y^{2},
$$

defined in a plane domain $\Omega$, can be reduced, by means of a suitable quasiconformal change of variables, to the canonical form

$$
d s^{2}=\Lambda\left(d u^{2}+d v^{2}\right), \Lambda \neq 0 \text { a.e. in } \Omega,
$$

provided that $a c-b^{2} \geq \triangle_{0}>0, a>0$, a.e. in $\Omega$, see, e.g., [7, p. 10-12]. This key result can be extended to every linear divergent operator of the form $\operatorname{div}[A(z) \nabla u(z)], z=x+i y$, for matrix function $A \in M^{2 \times 2}(\Omega)$. Namely, given such a matrix function $A$ and a quasiconformal mapping $\omega: \Omega \rightarrow G, \omega \in W_{\text {loc }}^{1,2}(\Omega)$ agreed with $A$, we have already seen by direct computation, that if the function $T$ and the entries of $A$ are sufficiently smooth, then

$$
\operatorname{div}[A(z) \nabla(T(\omega(z)))]=J_{\omega}(z) \triangle T(\omega(z)), z \in \Omega,
$$

see, e.g., $[15,16]$. Here $J_{\omega}(z)$ stands for the Jacobian of the mapping $\omega(z)$, e.g., $J_{\omega}(z)=\operatorname{det} D_{\omega}(z)$, where $D_{\omega}(z)$ is the Jacobian matrix of the mapping $\omega$ at the point $z \in \Omega$. Making use of the standard procedure, the regularity requirements in the equality (3.3) may be substantially weakened. The equality (3.4) below, that will be applied to the study of weak solutions to some semi-linear partial differential equations, can be viewed as a weak counterpart to the equality (3.3).

Proposition 3.1. Let $\Omega$ be a domain in $\mathbb{C}, A \in M^{2 \times 2}(\Omega)$ and $\omega: \Omega \rightarrow G$ be a quasiconformal mapping agreed with $A$. Then the equality

$$
\begin{aligned}
\int_{\Omega}\langle A(z) \nabla(T(\omega(z))), & \nabla \varphi(z)\rangle d m_{z} \\
& =\int_{\Omega}\left\langle D_{\omega}^{-1}(z) \nabla T(\omega(z)), \nabla \varphi(z)\right\rangle J_{\omega}(z) d m_{z}
\end{aligned}
$$

holds for every $T \in W_{\mathrm{loc}}^{1,2}(G)$ and for all $\varphi \in W_{0}^{1,2}(\Omega)$.

Proof. Assuming that $T \in W_{\mathrm{loc}}^{1,2}(G)$ and that $\omega: \Omega \rightarrow G$ is a quasiconformal mapping agreed with $A(z)$, we see, by Proposition 2.1, that $u:=T \circ \omega \in W_{\text {loc }}^{1,2}(\Omega)$. Since

$$
\nabla u(z)=D_{\omega}^{t}(z) \nabla T(\omega(z)),
$$


where $D_{\omega}^{t}(z)$ stands for the transpose matrix to $D_{\omega}(z)$, and $\omega$ satisfies the Beltrami equation (2.5), that can be written in the matrix form as

$$
A(z) D_{\omega}^{t}(z)=D_{\omega}^{-1}(z) J_{\omega}(z)
$$

we arrive successively to the required equality (3.4):

$$
\begin{aligned}
\int_{\Omega}\langle A(z) \nabla(T(\omega(z))), \nabla \varphi(z)\rangle d m_{z} \\
=\int_{\Omega}\left\langle A(z) D_{\omega}^{t}(z) \nabla T(\omega(z)), \nabla \varphi(z)\right\rangle d m_{z} \\
=\int_{\Omega}\left\langle D_{\omega}^{-1}(z) \nabla T(\omega(z)), \nabla \varphi(z)\right\rangle J_{\omega}(z) d m_{z}
\end{aligned}
$$

Remark 3.1. It is easy to see that if $T$ and $A$ are smooth and (3.4) holds, then (3.3) also holds. One can use the Green's formula as above, but in the opposite direction, to show that

$\int_{\Omega}\left\{\operatorname{div}[A(z) \nabla(T(\omega(z)))]-J_{\omega}(z) \triangle T(\omega(z))\right\} \varphi(z) d m_{z}=0, \forall \varphi \in C_{0}^{1}(\Omega)$.

Indeed, by Green's formula we have that

$$
\int_{\Omega}\langle A(z) \nabla(T(\omega(z))), \nabla \varphi(z)\rangle d m_{z}=-\int_{\Omega} \operatorname{div}[A(z) \nabla(T(\omega(z)))] \varphi(z) d m_{z} .
$$

On the other hand, applying again Green's formula after the change of variables $w=\omega(z)$, we get

$$
\begin{aligned}
\int_{\Omega} J_{\omega}(z) \triangle T(\omega(z)) \varphi(z) d m_{z} & \\
= & -\int_{\Omega}\left\langle\nabla T(\omega(z)),\left[D_{\omega}^{t}\right]^{-1}(z) \nabla \varphi(z)\right\rangle J_{\omega}(z) d m_{z}
\end{aligned}
$$

From the linear algebra it follows that

$$
\left\langle\nabla T(\omega(z)), J_{\omega}(z)\left[D_{\omega}^{t}\right]^{-1}(z) \nabla \varphi(z)\right\rangle=\left\langle J_{\omega}(z) D_{\omega}^{-1}(z) \nabla T(\omega(z)), \nabla \varphi(z)\right\rangle .
$$

Indeed, let $\omega(z)=a(z)+i b(z), z=x+i y$, then

$$
D_{\omega}=\left(\begin{array}{cc}
a_{x} & a_{y} \\
b_{x} & b_{y}
\end{array}\right)
$$




$$
\begin{aligned}
D_{\omega}^{t} & =\left(\begin{array}{cc}
a_{x} & b_{x} \\
a_{y} & b_{y}
\end{array}\right), \\
D_{\omega}^{-1} J_{\omega} & =\left(\begin{array}{cc}
b_{y} & -a_{y} \\
-b_{x} & a_{x}
\end{array}\right)
\end{aligned}
$$

and

$$
\left[D_{\omega}^{t}\right]^{-1} J_{\omega}=\left(\begin{array}{cc}
b_{y} & -b_{x} \\
-a_{y} & a_{x}
\end{array}\right) .
$$

Setting $\nabla T=(u, v)$, and $\nabla \varphi=(\xi, \eta)$, we get that

$$
\begin{aligned}
& \left\langle\nabla T, J_{\omega}\left[D_{\omega}^{t}\right]^{-1} \nabla \varphi\right\rangle=\left\langle(u, v),\left(b_{y} \xi-b_{x} \eta,-a_{y} \xi+a_{x} \eta\right)\right\rangle \\
& =u\left(b_{y} \xi-b_{x} \eta\right)+v\left(a_{x} \eta-a_{y} \xi\right), \\
& \left\langle J_{\omega} D_{\omega}^{-1} \nabla T, \nabla \varphi\right\rangle=\left\langle\left(b_{y} u-a_{y} v,-b_{x} u+a_{x} v\right),(\xi, \eta)\right\rangle \\
& =u\left(b_{y} \xi-b_{x} \eta\right)+v\left(a_{x} \eta-a_{y} \xi\right),
\end{aligned}
$$

and we arrive to formula (3.10).

Thus,

$$
\begin{aligned}
& \int_{\Omega} J_{\omega}(z) \triangle T(\omega(z)) \varphi(z) d m_{z} \\
&=-\int_{\Omega}\left\langle D_{\omega}^{-1}(z) \nabla T(\omega(z)), \nabla \varphi(z)\right\rangle J_{\omega}(z) d m_{z}
\end{aligned}
$$

Finally, since $C_{0}^{1}(\Omega)$ is dense in $W_{0}^{1,2}(\Omega)$, we come to the equality (3.3) for almost all points in $\Omega$. However, since all functions are smooth, the equation holds at every point.

\section{Semi-linear model elliptic equations}

Let $\Omega$ be a bounded domain in $\mathbb{C}$ and let $f: \mathbb{R} \rightarrow \mathbb{R}$ be a continuous function. In this section we study a model semi-linear equation

$$
\operatorname{div}[A(z) \nabla u(z)]=f(u(z)), \quad z \in \Omega
$$

as well as its Laplace's counterpart:

$$
\triangle T(w)=J(w) f(T(w)), \quad w \in G=\omega(\Omega),
$$

where $\omega: \Omega \rightarrow G$ is a quasiconformal mapping agreed with $A(z)$ and $J(w)$ stands for the Jacobian of the inverse mapping $\omega^{-1}: G \rightarrow \Omega$. 
Under a weak solution to the equation (4.1) we understand a function $u \in C \cap W_{\text {loc }}^{1,2}(\Omega)$ such that

$$
\int_{\Omega}\langle A(z) \nabla u(z), \nabla \varphi(z)\rangle d m_{z}+\int_{\Omega} f(u(z)) \varphi(z) d m_{z}=0 \forall \varphi \in C \cap W_{0}^{1,2}(\Omega) .
$$

The equation (4.2) contains a weight function $J(w) \in L_{\text {loc }}^{1}(G)$. In spite of this, we may define a notion of a weak solution to the equation (4.2) in the similar way.

We say that $T$ is a weak solution to the equation (4.2) if $T \in C \cap$ $W_{\mathrm{loc}}^{1,2}(G)$ and

$$
\begin{aligned}
\int_{G}\langle\nabla T(w), \nabla \psi(w)\rangle d m_{w} & \\
& +\int_{G} J(w) f(T(w)) \psi(w) d m_{w}=0 \quad \forall \psi \in C \cap W_{0}^{1,2}(G) .
\end{aligned}
$$

Since $J(w)$ is the Jacobian of the mapping $\omega^{-1}(w)$, it is easy to verify, by performing the change of variable by the formula $w=\omega(z)$, that the second integral in (4.4) is well-defined. Here we again made use of the fact that the composed mapping $u(z)=T(\omega(z))$ is in $C \cap W_{\text {loc }}^{1,2}(\Omega)$ if $T \in C \cap W_{\text {loc }}^{1,2}(G)$ and $\omega$ is quasiconformal.

The existence of weak solutions to the equations (4.1) and (4.2), under specified conditions on the right hand sides, as well as fundamental properties of solutions can be found, e.g. in [24], see also [22].

The following Factorization Theorem is a principal result of this paper.

Theorem 4.1. Let $\Omega$ be a domain in $\mathbb{C}, A \in M^{2 \times 2}(\Omega)$ and let $f: \mathbb{R} \rightarrow \mathbb{R}$ be a continuous function. Then every weak solution $u$ of the semi-linear equation

$$
\operatorname{div}[A(z) \nabla u(z)]=f(u(z)), \quad z \in \Omega,
$$

can be represented as the composition

$$
u(z)=T(\omega(z))
$$

where $\omega: \Omega \rightarrow G$ is a quasiconformal mapping agreed with $A$ and $T$ is a weak solution to the equation

$$
\triangle T(w)=J(w) f(T(w)), w \in G .
$$

Here $J(w)$ stands for the Jacobian of the inverse mapping $\omega^{-1}(w)$. 
Proof. Let $u$ be a weak solution of the semi-linear equation (4.5) and $T=u \circ \omega^{-1}$. Then by Proposition 2.1 $T \in C \cap W_{\text {loc }}^{1,2}(G)$ and we have that

$$
\int_{\Omega}\langle A(z) \nabla(T(\omega(z))), \nabla \varphi(z)\rangle d m_{z}+\int_{\Omega} f(T(\omega(z))) \varphi(z) d m_{z}=0
$$

for all $\varphi \in C \cap W_{0}^{1,2}(\Omega)$. Next, by Proposition 3.1,

$$
\begin{aligned}
\int_{\Omega}\langle A(z) \nabla(T(\omega(z))), & \nabla \varphi(z)\rangle d m_{z} \\
& =\int_{\Omega}\left\langle D_{\omega}^{-1}(z) \nabla T(\omega(z)), \nabla \varphi(z)\right\rangle J_{\omega}(z) d m_{z},
\end{aligned}
$$

and therefore

$$
\int_{\Omega}\left\langle J_{\omega}(z) D_{\omega}^{-1}(z) \nabla T(\omega(z)), \nabla \varphi(z)\right\rangle d m_{z}+\int_{\Omega} f(T(\omega(z))) \varphi(z) d m_{z}=0
$$

for all $\varphi \in C \cap W_{0}^{1,2}(\Omega)$.

Given an arbitrary function $\psi(w) \in C \cap W_{0}^{1,2}(G)$, we can set in (4.8) and (4.9) $\varphi(z)=\psi(\omega(z))$, because, by Proposition 2.1, such $\varphi \in$ $C \cap W_{0}^{1,2}(\Omega)$. Performing in (4.10) the change of variable by the formula $z=\omega^{-1}(w)$, we obtain

$$
\begin{array}{r}
\int_{G}\left\langle J_{\omega}\left(\omega^{-1}(w)\right) D_{\omega}^{-1}\left(\omega^{-1}(w)\right) \nabla T(w), D_{\omega}^{t}\left(\omega^{-1}(w)\right) \nabla \psi(w)\right\rangle J(w) d m_{w} \\
+\int_{G} J(w) f(T(w)) \psi(w) d m_{w}=0 .
\end{array}
$$

Since, by elementary algebraic arguments,

$$
\begin{gathered}
\left\langle J_{\omega}\left(\omega^{-1}(w)\right) D_{\omega}^{-1}\left(\omega^{-1}(w)\right) \nabla T(w), D_{\omega}^{t}\left(\omega^{-1}(w)\right) \nabla \psi(w)\right\rangle \\
=J_{\omega}\left(\omega^{-1}(w)\right)\langle\nabla T(w), \nabla \psi(w)\rangle,
\end{gathered}
$$

and

$$
J_{\omega}\left(\omega^{-1}(w)\right)=1 / J(w),
$$

we see that the identity

$$
\int_{G}\langle\nabla T(w), \nabla \psi(w)\rangle d m_{w}+\int_{G} J(w) f(T(w)) \psi(w) d m_{w}=0
$$

holds for all $\psi \in C \cap W_{0}^{1,2}(G)$. Thus, $T$ is a weak solution to the equation (4.7). 
Remark 4.1. Since the arguments given above are invertible, we see that if $T$ is a weak solution to the equation (4.7), then the function $u(z)=T(\omega(z))$ is a weak solution to the semi-linear equation (4.5).

Assuming that the function $f$ is non-negative, we arrive at the following statement.

Corollary 4.1. Let $\Omega$ be a domain in $\mathbb{C}, A \in M^{2 \times 2}(\Omega)$ and let $f: \mathbb{R} \rightarrow \mathbb{R}$ be a continuous function. If $f(u) \geq 0$, then every weak solution $u$ of the semi-linear equation

$$
\operatorname{div}[A(z) \nabla u(z)]=f(u(z)), \quad z \in \Omega,
$$

can be represented as the composition

$$
u(z)=T(\omega(z)),
$$

where $\omega: \Omega \rightarrow G$ is quasiconformal mapping agreed with $A$ and $T$ is a subharmonic function, being a weak solution of the equation

$$
\triangle T=J(w) f(T(w)) \text { in } G .
$$

Here $J(w)$ being a weak solution of the Jacobian of the inverse mapping $\omega^{-1}(w)$.

Among the quasiconformal mappings $\omega: \Omega \rightarrow G$ there are a variety of the so-called volume-preserving maps, for which $J_{\omega}(z) \equiv 1, z \in \Omega$. Many examples of such maps will be given in the next section. The following statement may have of independent interest.

Corollary 4.2. Let $A \in M^{2 \times 2}(\Omega)$ be a matrix function that generates a volume-preserving quasiconformal mapping $\omega: \Omega \rightarrow G$. Then every weak solution $u$ of the semi-linear equation

$$
\operatorname{div}[A(z) \nabla u(z)]=f(u(z)), \quad z \in \Omega,
$$

can be represented as the composition

$$
u(z)=T(\omega(z)),
$$

where $T$ is a weak solution to the equation

$$
\triangle T=f(T(w)), \quad \text { a.e. in } G .
$$


Remark 4.2. By the Measurable Riemann mapping theorem, see e.g. Theorem V.B.3 in [1] and Theorem V.1.3 in [25], given $\mu(z), z \in \Omega$, agreed with the matrix function $A \in M^{2 \times 2}(\Omega)$, there exists a quasiconformal mapping $\omega: \Omega \rightarrow G$ with the complex dilatation $\mu$ given by (2.6). Here if $\Omega$ is finitely connected, then $G$ is either the canonical circular domain, or the complex plane, see e.g. Theorem V.6.2 in [12]. It allows us to choose in the Factorization Theorem 4.1 as the domain $G$ one from the above canonical domains. If $\Omega$ is simply connected with a nondegenerate boundary then we can set $G=\mathbb{D}$. The latter makes it possible to remove the restrictions on the regularity of the boundary in the study of boundary value problems for the equation (4.7), see Section 8 .

\section{Some Applications}

We start with a few examples of the application of Theorem 4.1 to the study of boundary blow-up solutions for some classical model semi-linear elliptic equations.

Let $\Omega$ be a bounded domain in $\mathbb{C}$ and let $\partial \Omega$ denote its boundary. In this section we study the problem

$$
\begin{aligned}
& \operatorname{div}[A(z) \nabla u(z)]=f(u(z)), \\
& u(z) \rightarrow \infty \quad \text { as } \quad d(z):=\operatorname{dist}(z, \partial \Omega) \rightarrow 0,
\end{aligned}
$$

as well as its Laplace counterpart:

$$
\begin{gathered}
\triangle u(z)=f(u(z)), \\
u(z) \rightarrow \infty \quad \text { as } \quad d(z):=\operatorname{dist}(z, \partial \Omega) \rightarrow 0 .
\end{gathered}
$$

Solutions to these problems are called boundary blow-up solutions, or large solution.

The existence of a large solution to (5.3) is related to the existence of a maximal solution $\tilde{u}$ of (5.3) in $\Omega$, which in turn depends on the so called Keller-Osserman condition, see [20] and [29]. For example, in the paper [20] a simple upper bound was obtained for any solution, in any number of variables, of semi-linear equation (5.3). The bound depends on the function $f$ which must be positive and satisfy the well-known Keler-Osserman condition. Recall that a function $f \in C\left(\mathbb{R}_{+}\right)$satisfies the Keler-Osserman condition if there exists a positive non-decreasing function $h$ such that

$$
f(t) \geq h(t), \forall t \in \mathbb{R}_{+} \text {and } \int_{t_{0}}^{\infty}\left\{\int_{0}^{t} h(s) d s\right\}^{-1 / 2} d t<\infty \text { for all } t_{0}>0 .
$$


It is known that if $f$ is non-decreasing and satisfies the Keller-Osserman condition, then a large solution exists in every bounded smooth domain. Uniqueness in smooth domains was established under some additional conditions on $f$, see e.g. [3]. It is easy to check that the functions $f(t)=e^{t}$ and $f(t)=t^{p}, p>1$, satisfy (5.5). The Liouville-Bieberbach semi-linear equation

$$
\triangle u=e^{u},
$$

as far as we know, was first investigated by Bieberbach in his pioneering work [5] related to the study of automorphic functions in the plane. It is this work has stimulated numerous studies in the field of semi-linear differential equations in $R^{n}, n \geq 1$, and the equation (5.6) continues to play the role of one of the fundamental model equations of the theory. It is important to note that in simply connected planar domains $\Omega$ the large solutions for the equation (5.6) are represented explicitly by means of the conformal map $\omega: \Omega \rightarrow \mathbb{D}:=\{z \in \mathbb{C}:|z|<1\}$ :

$$
u(z)=\log \frac{8\left|\omega^{\prime}(z)\right|^{2}}{\left(1-|\omega(z)|^{2}\right)^{2}} .
$$

Let us also remark that, given an arbitrary analytic function $\omega(z)$ in $\Omega$, the formula (5.7) generates a solution, generally speaking with singularities, of the equation (5.6).

For the model case of equation

$$
\Delta u=e^{a u}, \quad a>0,
$$

the following result holds, see [23, Theorem 5.3.7].

Theorem 5.1. Let $\Omega$ be a bounded domain in $\mathbb{C}$ such that $\Omega=\operatorname{Int} \bar{\Omega}$. Then there exists one and only one blow-up solution to (5.8).

The Liouville-Bieberbach semi-linear equation is one of the principal model equations in the theory of non-linear partial differential equations and their applications, see e.g. $[23,26,27]$ and the references therein. Note that the equation appears also as a model one in problems of differential geometry in relation with existence of surfaces of negative Gaussian curvature [35] and in studying the equilibrium of a charged gas.

In the general case for $|\mu(z)| \leq k<1$ the solution of the Beltrami equation can be written as an infinite series of singular integral transforms of Hilbert and Cauchy type for complex dilatations, see, e.g. [7, p. 33]. Here we give the explicit solutions of the Beltrami equation for the cases where the complex dilatation is a measurable function that depends on a 
single real variable $x=\operatorname{Re} z, y=\operatorname{Im} z, \arg z$ or $|z|$, see [7, §5.10], [17]. We make use of the corresponding explicit formulae to write down a number of explicit solutions for the counterpart (5.1) of the Liouville-Bieberbach equation in the unit disk and the upper half-plane.

We start with the following statement, see, e.g., [7, p. 82].

Proposition 5.1. Let the complex dilatation $\mu(z)$ has the form

$$
\mu(z)=k(|z|) \frac{z}{\bar{z}}
$$

where $k(\tau): \mathbb{R} \rightarrow \mathbb{C}$ is a measurable function, $\|k\|_{\infty}<1$. Then the formula

$$
\omega(z)=\frac{z}{|z|} \exp \left\{-\int_{|z|}^{1} \frac{1+k(\tau)}{1-k(\tau)} \frac{d \tau}{\tau}\right\}
$$

represents a unique quasiconformal mapping of the unit disk onto itself with the complex dilatation $\mu$ and the normalizations $\omega(0)=0$ and $\omega(1)=1$.

Analyzing formula (5.10), we arrive at the following statement.

Corollary 5.1. Let $\mathbb{D}$ be the unit disk in the complex plane $\mathbb{C}$ centered at the origin and let the matrix function $A(z)$ be generated by the Beltrami coefficient

$$
\mu(z)=k(|z|) \frac{z}{\bar{z}}
$$

in accordance with formula (2.8) where

$$
k(t)=\nu^{2}(t) \pm i \nu(t) \sqrt{1-\nu^{2}(t)}
$$

and $\nu(t), 0 \leq t<1$, stands for an arbitrary measurable real-valued function such that $|\nu(t)| \leq q<1$. Then there exists one and only one boundary blow-up solution to the semi-linear equation

$$
\operatorname{div}[A(z) \nabla u]=e^{u}, \quad z \in \mathbb{D},
$$

which is written explicitly by the Liouville-Bieberbach formula

$$
u(z)=\log \frac{8}{\left(1-|z|^{2}\right)^{2}} .
$$


Proof. Let $A(z)$ be a matrix function generated by the complex Beltrami coefficient (5.11), satisfying (5.12). By the Measurable Riemann mapping theorem, see e.g. Theorem V.B.3 in [1] and Theorem V.1.3 in [25], there exists the unique normalized quasiconformal self-homeomorphism $\omega$ of the unit disk $\mathbb{D}$ with the Beltrami coefficient $\mu(z)=k(|z|) z / \bar{z}$ and this homeomorphism, by Proposition 5.1, is written explicitly,

$$
\omega(z)=\frac{z}{|z|} \exp \left\{-\int_{|z|}^{1} \frac{1+k(t)}{1-k(t)} \frac{d t}{t}\right\} .
$$

We see that

$$
\begin{aligned}
& \frac{\omega_{\bar{z}}}{\omega}=\frac{k}{1-k} \frac{1}{\bar{z}}, \\
& \frac{\omega_{z}}{\omega}=\frac{1}{1-k} \frac{1}{z}
\end{aligned}
$$

and therefore, for the Jacobian $J_{\omega}(z)=\left|\omega_{z}\right|^{2}-\left|\omega_{\bar{z}}\right|^{2}$ we have

$$
J_{\omega}(z)=\frac{1-|k|^{2}}{|1-k|^{2}} \frac{|\omega|^{2}}{|z|^{2}} .
$$

In order to apply Theorem 4.2 , we have to verify that $J_{\omega}(z) \equiv 1$ and $|\omega(z)|=|z|$ for $z \in \mathbb{D}$. Indeed, noting that $\operatorname{Re} k=|k|^{2}$ and substituting $k(t)$ given by formula (5.12) into (5.15) we see that $|\omega(z)|=|z|, z \in \mathbb{D}$. The later implies that $J_{\omega}(z) \equiv 1$.

Making use of Corollary 4.2, we see that $u(z)=T(\omega(z))$ and $T$ satisfies the Liouville-Bieberbach equation

$$
\triangle T=e^{T} \text { in } \mathbb{D} .
$$

By the mentioned above Bieberbach's result and referring also to Theorem 5.3.7 from [23], we see that the unique boundary blow-up solution to the equation (5.17) in the unit disk $\mathbb{D}$ is given by the explicit formula

$$
T(w)=\log \frac{8}{\left(1-|w|^{2}\right)^{2}} .
$$

Since $|\omega(z)|=|z|$ for $z \in \mathbb{D}$, the required solution to the equation (5.13) also has the explicit representation

$$
u(z)=\log \frac{8}{\left(1-|z|^{2}\right)^{2}} .
$$


Thus, there is an infinite set of matrix functions $A(z)$ such that the corresponding anisotropic semi-linear equations of the form (5.13) have a solution defined by the explicit formula (5.14).

Let us give a nontrivial example, based on the well-known logarithmic spiral quasiconformal mapping

$$
\omega(z)=z e^{2 i \log |z|}
$$

which plays important role in the study of different problems of contemporary analysis, see, e.g., [7, §13.2], [13,14]. This function $\omega$ maps the unit disk $\mathbb{D}$ onto itself and transforms radial lines into spirals, infinitely winding about the origin, and it is just the volume preserving. The mapping $\omega$ satisfies the Beltrami equation with

$$
\mu(z)=\frac{\omega_{\bar{z}}}{\omega_{z}}=\frac{1}{2}(1+i) \frac{z}{\bar{z}}
$$

and the Jacobian $J_{\omega}(z)=\left|\omega_{z}(z)\right|^{2}-\left|\omega_{\bar{z}}(z)\right| \equiv 1$. We see that $\mu$ corresponds to (5.12) with $\nu(t) \equiv 1 / \sqrt{2}$. Since

$$
\operatorname{Re} \mu(z)=\frac{x^{2}-y^{2}-2 x y}{2\left(x^{2}+y^{2}\right)}, \operatorname{Im} \mu(z)=\frac{x^{2}-y^{2}+2 x y}{2\left(x^{2}+y^{2}\right)}, z=x+i y,
$$

we see that $\mu$ generates by the formula (2.8) the matrix function $A_{s p}(x, y)$ with the following entries:

$$
\begin{gathered}
a_{11}=\alpha=\frac{|1-\mu|^{2}}{1-|\mu|^{2}}=3-2 \frac{x^{2}-y^{2}-2 x y}{\left(x^{2}+y^{2}\right)}, \\
a_{22}=\delta=\frac{|1+\mu|^{2}}{1-|\mu|^{2}}=3+2 \frac{x^{2}-y^{2}-2 x y}{\left(x^{2}+y^{2}\right)}, \\
a_{12}=a_{21}=\beta=\frac{-2 \operatorname{Im} \mu}{1-|\mu|^{2}}=-2 \frac{x^{2}-y^{2}+2 x y}{\left(x^{2}+y^{2}\right)} .
\end{gathered}
$$

By Corollary 5.1 the semi-linear equation

$$
\operatorname{div}\left[A_{s p}(x, y) \nabla u\right]=e^{u}, \quad z \in \mathbb{D},
$$

must have the function

$$
u(x, y)=-2 \log \left(1-x^{2}-y^{2}\right)+\log 8
$$

as the blow-up solution in the disk $\mathbb{D}$. Let us verify this conclusion by means of direct computation. 
We see that

$$
u_{x}=\frac{4 x}{1-x^{2}-y^{2}}, u_{y}=\frac{4 y}{1-x^{2}-y^{2}}
$$

and therefore

$$
\alpha u_{x}+\beta u_{y}=4 \frac{x+2 y}{1-x^{2}-y^{2}}, \quad \beta u_{x}+\delta u_{y}=4 \frac{y-2 x}{1-x^{2}-y^{2}} .
$$

Then

$\operatorname{div}\left(A_{s p}(x, y) \nabla u\right)=4\left(\alpha u_{x}+\beta u_{y}\right)_{x}^{\prime}+4\left(\beta u_{x}+\delta u_{y}\right)_{y}^{\prime}=\frac{8}{\left(1-x^{2}-y^{2}\right)^{2}}=e^{u}$,

and thus we successfully complete the verification.

Note also that the matrix function $A_{s p}$ in the polar coordinates $z=$ $r e^{i \varphi}$ has the form

$$
A=\left(\begin{array}{cc}
3-2 \sqrt{2} \cos (2 \varphi+\pi / 4) & -2 \sqrt{2} \sin (2 \varphi+\pi / 4) \\
-2 \sqrt{2} \sin (2 \varphi+\pi / 4) & 3+2 \sqrt{2} \cos (2 \varphi+\pi / 4)
\end{array}\right) .
$$

Corollary 5.2. Let $\Omega$ be the annulus $r<|z|<1$ in the complex plane $\mathbb{C}$ and let the matrix function $A(z)$ be generated by the Beltrami coefficient

$$
\mu(z)=k(|z|) \frac{z}{\bar{z}}
$$

in accordance with formula (2.8) where

$$
k(t)=\nu^{2}(t) \pm i \nu(t) \sqrt{1-\nu^{2}(t)}
$$

and $\nu(t), 0 \leq t<1$ stands for an arbitrary measurable function. If $|\nu(t)| \leq q<1$, then there exists one and only one boundary blow-up solution to the classical model semi-linear equation

$$
\operatorname{div}[A(z) \nabla u]=e^{u}, \quad \text { in the annulus } r<|z|<1
$$

and which is written explicitly by the formula

$$
u(z)=\log \frac{2 \pi^{2}}{|z|^{2}\left(\log ^{2} r\right) \cdot \sin ^{2}\left(\frac{\pi}{\log r} \log |z|\right)} .
$$


Proof. Arguing in the same way as in the proof of Corollary 5.1, we see that $u(z)=T(\omega(z)), r<|z|<1$, where quasiconformal self-homeomorphism $\omega(z)$ of the annulus is given by (5.15), (note that under the condition (5.23) $|\omega(z)|=|z|)$, and $T$ is the unique boundary blow-up solution to the semi-linear equation

$$
\triangle T=e^{T}
$$

in the annulus $r<|w|<1$. By Bieberbach's result, the unique boundary blow-up solution to the equation (5.26) is given by the formula

$$
T(\omega)=\log \frac{8\left|F^{\prime}(\omega)\right|^{2}}{\left(1-|F(\omega)|^{2}\right)^{2}}
$$

where $F(\omega)$ stands for a conformal mapping of the annulus $r<|\omega|<$ 1 onto the unit disk. It remains to find the corresponding conformal mapping $F$. We see that:

1) $w=\log \omega$ maps the annulus onto the strip $\log r<\operatorname{Re} w<0$;

2) $\zeta=-i e^{\frac{\pi}{\log r} i w}$ maps the strip onto the right half-plane;

3) $t=(\zeta-1) /(\zeta+1)$ maps the right half-plane onto the unit disk. Composing the above mappings we get the required formula

$$
F(\omega)=\frac{i e^{\frac{\pi}{\log r} i \log \omega}+1}{i e^{\frac{\pi}{\log r} i \log \omega}-1} .
$$

Next, if we set $\tau(\omega)=i e^{\frac{\pi}{\log r} i \log \omega}$, then we see that

$$
\left(1-|F(\omega)|^{2}\right)^{2}=\frac{16 \operatorname{Re}^{2} \tau(\omega)}{|\tau-1|^{4}}
$$

where

$$
\operatorname{Re} \tau(\omega)=-|\tau| \sin \left(\frac{\pi}{\log r} \log |\omega|\right) .
$$

On the other hand,

$$
\left|F^{\prime}(\omega)\right|^{2}=\frac{4}{|\tau-1|^{4}}\left|\tau^{\prime}(\omega)\right|^{2}=\frac{4}{|\tau-1|^{4}}|\tau|^{2} \frac{\pi^{2}}{\log ^{2} r} \frac{1}{|\omega|^{2}} .
$$

Thus

$$
T(\omega)=\log \frac{2 \pi^{2}}{|\omega|^{2}\left(\log ^{2} r\right) \cdot \sin ^{2}\left(\frac{\pi}{\log r} \log |\omega|\right)}
$$

and since $|\omega(z)|=|z|$, we complete the proof of Corollary 5.2. 
Making use of the limit in (5.25) as $r \rightarrow 0$ we get the following result which may have of independent interest.

Corollary 5.3. The semi-linear equation

$$
\operatorname{div}[A(z) \nabla u]=e^{u}
$$

for each matrix function A from Corollary 5.2 as well as the LiouvilleBieberbach equation

$$
\triangle u=e^{u}
$$

admit the following boundary blow-up solution in the punctured unit disk $0<|z|<1$

$$
u(z)=\log \frac{2}{|z|^{2} \log ^{2}|z|} .
$$

Remark 5.1. The approach given above to the construction of a boundary blow-up solution to the Liouville-Bieberbach equation in the unit disk $\mathbb{D}$ with a singularity at the origin can be extended to the case of a finite number of singular points $z_{k},\left|z_{k}\right|<1, k=1,2, \ldots, n$. Indeed, let $r>0$ be such that all the disks $d_{k}=\left\{z:\left|z-z_{k}\right| \leq r\right\}$ belong to $\mathbb{D}$ and do not intersect each other. Denote by $F_{r}(z)$ a conformal mapping of the circular multi-connected domain $\mathbb{D} \backslash \cup_{k=1}^{n} d_{k}$ onto the unit disc $\mathbb{D}$, see Theorem VI.1 in [12] due to Poincare. Then the required solution with prescribed singularities at the points $z_{k}$ is given by

$$
u(z)=\lim _{r \rightarrow 0} \log \frac{8\left|F_{r}^{\prime}(z)\right|^{2}}{\left(1-\left|F_{r}(z)\right|^{2}\right)^{2}} .
$$

In order to give more examples of applications of our Factorization Theorem and its corollaries, recall the following statement, which provides us with the explicit solutions of the Beltrami equation for the cases where the complex dilatation $\mu$ is a measurable function that depends on a single real variable $x=\operatorname{Re} z$ or $y=\operatorname{Im} z$, see, [7, p. 78], [17].

Proposition 5.2. Let $\mu: \mathbb{C} \rightarrow \mathbb{D}$ be an arbitrary measurable function with $\|\mu\|_{\infty} \leq q<1$ that depends on $x=\operatorname{Re} z$ only and let

$$
\varphi(x)=\int_{0}^{x} \frac{1+\mu(t)}{1-\mu(t)} d t .
$$

Then the formula

$$
\omega(z)=\varphi(x)+i y
$$


represents a unique quasiconformal mapping of $\overline{\mathbb{C}}$ onto itself with complex dilatation $\mu$ and normalizations:

$$
\omega(0)=0, \quad \omega(i)=i, \quad \omega(\infty)=\infty .
$$

Indeed, from formulas (5.36) and (5.37) it follows that

$$
K^{-1}\left|x_{1}-x_{2}\right| \leq\left|\omega\left(z_{1}\right)-\omega\left(z_{2}\right)\right| \leq K\left|z_{1}-z_{2}\right| .
$$

Thus, $\omega$ is a lipschitz homeomorphism of the plane and hence ACL. The normalization (5.38) is obvious. Furthermore,

$$
\begin{aligned}
\omega_{x}=\varphi^{\prime}(x) & =\frac{1+\mu(x)}{1-\mu(x)}, \\
\omega_{y} & =i .
\end{aligned}
$$

Therefore,

$$
\begin{aligned}
& \omega_{\bar{z}}=\frac{1}{2}\left(\omega_{x}+i \omega_{y}\right)=\frac{\mu(x)}{1-\mu(x)}, \\
& \omega_{z}=\frac{1}{2}\left(\omega_{x}-i \omega_{y}\right)=\frac{1}{1-\mu(x)},
\end{aligned}
$$

and hence $\omega$ satisfies the Beltrami equation

$$
\omega_{\bar{z}}=\mu(x) \omega_{z} .
$$

The Jacobian of $\omega$

$$
J_{\omega}(z)=\frac{1-|\mu(x)|^{2}}{|1-\mu(x)|^{2}} \geq K^{-1}>0
$$

is positive, that is, $\omega$ is orientation-preserving. This completes the proof of Proposition 5.2.

Remark 5.2. From

$$
\begin{gathered}
u=\operatorname{Re} \omega(z)=\operatorname{Re} \varphi(x), \\
v=\operatorname{Im} \omega(z)=y+\operatorname{Im} \varphi(x),
\end{gathered}
$$

we conclude that $\omega$ maps vertical lines onto vertical lines without contractions or dilations, and the imaginary axis is mapped identically onto itself, since $\varphi(0)=0$. It is easy to show that these geometric properties characterize the class of quasiconformal mappings in question, because

$$
\mu(z)=\frac{\varphi^{\prime}(x)-1}{\varphi^{\prime}(x)+1}
$$

depends only on $x$. 
Corollary 5.4. Suppose that the complex dilatation $\nu(z)$ of a quasiconformal mapping $g(z)$ of the extended complex plane, keeping the points $0,1, \infty$, depends only on $y=\operatorname{Im} z$. Then

$$
g(z)=x+i \psi(y)
$$

where

$$
\psi(y)=\int_{0}^{y} \frac{1-\nu(i t)}{1+\nu(i t)} d t .
$$

Indeed, let

$$
\omega=\mathcal{A} \circ g \circ \mathcal{A}^{-1}
$$

where

$$
\mathcal{A}(\zeta)=e^{i \frac{\pi}{2}} \zeta=i \zeta
$$

is a counter-clockwise rotation by the angle $\pi / 2$. Then $\omega(0)=0, \omega(i)=i$, $\omega(\infty)=\infty$, and the complex dilatation of $\omega$,

$$
\mu(z)=-\nu(-i z)
$$

depends only on $x=\operatorname{Re} z$. Thus, from (5.36), (5.37), and (5.51) we obtain (5.49) and (5.50).

Let us return to the study of the blow-up solutions to the LiouvilleBiberbach type equation defined in the right half-plane.

Corollary 5.5. Let $H^{+}$be the right half-plane $\{z: \operatorname{Re} z>0\}$ in the complex plane $\mathbb{C}$ and let the matrix function $A(z)$ be generated by the formula (2.8) with the Beltrami coefficient

$$
\mu(x)=\nu^{2}(x) \pm i \nu(x) \sqrt{1-\nu^{2}(x)}
$$

where $\nu(x), x \in \mathbb{R}$, stands for an arbitrary measurable real-valued function, such that $|\nu(x)| \leq q<1$. Then there exist boundary blow-up solutions to the semi-linear equation

$$
\operatorname{div}[A(z) \nabla u]=e^{u}, \quad z \in H^{+},
$$

and which are written explicitly:

$$
\begin{gathered}
u(z)=\log \frac{2}{x^{2}}, \quad z=x+i y, \\
u(z)=\log 8 \lambda^{2}-2 \lambda x-2 \log \left(1-e^{-2 \lambda x}\right), \quad \lambda>0 .
\end{gathered}
$$


Proof. We prove only that the function $u(z)$, given by the formula (5.56) solves the equation (5.55). Let $A(z)$ be a matrix function generated by the complex Beltrami coefficient $\mu$, satisfying (5.54). Since $\mu$ depends on $x$ only, then formulae (5.36), (5.37) represent a unique quasiconformal mapping $w=\omega(z)$ of the right half-plane $H^{+}$onto itself with complex dilatation $\mu$ normalized by $\omega(0)=0, \omega(i)=i, \omega(\infty)=\infty$.

In order to apply Corollary 4.2 , we have first to verify that $J_{\omega}(z) \equiv 1$. Indeed, in our case $\operatorname{Re} \mu=|\mu|^{2}$ and formula (5.45) implies that $J_{\omega}(z) \equiv 1$. Moreover, $\operatorname{Re} \omega(z)=\operatorname{Re} \varphi(x)=x$. By Corollary 4.2 a weak solution $u$ of the semi-linear equation

$$
\operatorname{div}[A(z) \nabla u(z)]=e^{u}, \quad z \in H^{+},
$$

is represented as the composition

$$
u(z)=T(\omega(z)),
$$

where $T$ is a weak solution to the equation

$$
\triangle T(w)=e^{T(w)}, \quad \text { in } H^{+} .
$$

Since the function

$$
F(w)=\frac{w-1}{w+1}
$$

is a conformal mapping of $\mathrm{H}^{+}$onto the unit disk $\mathbb{D}$, we see that the function

$$
T(w)=\log \frac{8\left|F^{\prime}(w)\right|^{2}}{\left(1-|F(w)|^{2}\right)^{2}}=-2 \log \operatorname{Re} w+\log 2
$$

gives us a blow-up solution to the equation (5.60) in $H^{+}$. Now, by formula (5.59), we have that the first required solution has the form

$$
u(z)=T(\omega)=-2 \log \operatorname{Re} \omega(z)+\log 2=-2 \log \operatorname{Re} x+\log 2 .
$$

In order to get the second solution, we have to note that the function

$$
F(w)=e^{-\lambda w}, \quad \lambda>0,
$$

is also conformal mapping of $H^{+}$onto the punctured unit disk $\mathbb{D} \backslash\{0\}$. Repeating the above arguments and taking into account that $\operatorname{Re} \omega(z)=$ $x$, we arrive to solution (5.57)

$$
u(z)=T(\omega)=\log \frac{8 \lambda^{2}\left|e^{-\lambda \omega(z)}\right|^{2}}{\left(1-\left|e^{-\lambda \omega(z)}\right|^{2}\right)^{2}}=\log 8 \lambda^{2}-2 \lambda x-2 \log \left(1-e^{-2 \lambda x}\right) .
$$


As an example, one can take the matrix function $A$ with the following constant entries $a_{11}=1, a_{12}=-2, a_{22}=5$. Then $\mu(z)=(1+i) / 2$ and $\omega(z)=x+i(y+2 x)$. It is easy to verify by straightforward computation that the functions (5.56), (5.57) solve the equation (5.55). In the general case the admissible matrices in Corollary 5.3 have the entries: $a_{11}=1$, $a_{12}= \pm 2 \nu(x) / \sqrt{1-\nu^{2}(x)}, a_{22}=\left(1+3 \nu^{2}(x)\right) /\left(1-\nu^{2}(x)\right)$. The particular case considered above corresponds to the function $\nu(x)=1 / \sqrt{2}$.

Similar results can be given for the upper half-plane on the base of Corollary 5.4.

\section{Free boundary}

The very important applied effect of the "dead zone" for solutions of some partial differential equations, see e.g. [9], the Introduction and §1, is that a solution of the corresponding differential equation vanishes on some nonempty open proper subset of the domain of its definition. For example, it is well-known that a solution of the semi-linear equation

$$
\triangle u=u^{q}
$$

may have the "dead zone" only when $0<q<1$, see e.g. [9, p. 15].

We confine ourselves to only one result in this respect, which is a simple consequence of Proposition 5.2 and Corollary 4.2.

Theorem 6.1. Let $\mathbb{C}$ be the complex plane and let the matrix function $A(z), z=x+i y$, be generated by the Beltrami coefficient

$$
\mu(x)=\nu^{2}(x) \pm i \nu(x) \sqrt{1-\nu^{2}(x)},
$$

that is

$$
A(z)=\left(\begin{array}{cc}
1 & \mp \frac{2 \nu(x)}{\sqrt{1-\nu^{2}(x)}} \\
\mp \frac{2 \nu(x)}{\sqrt{1-\nu^{2}(x)}} & \frac{1+3 \nu^{2}(x)}{1-\nu^{2}(x)}
\end{array}\right),
$$

where $\nu(x), x \in \mathbb{R}$, stands for an arbitrary measurable real-valued function, such that $|\nu(x)| \leq k<1$. Then the semi-linear equation

$$
\operatorname{div}[A(z) \nabla u]=u^{q}, \quad 0<q<1, z \in \mathbb{C},
$$

has in the complex plane the following solution with the "dead zone":

$$
u(x, y)=\left\{\begin{array}{cc}
\gamma\left(y \pm \int_{0}^{x} \frac{2 \nu(t)}{\sqrt{1-\nu^{2}(t)}} d t\right)^{\frac{2}{1-q}}, & \text { if } y>\varphi_{ \pm}(x), x \in \mathbb{R}, \\
0 & \text { if } x \leq \varphi_{ \pm}(x) .
\end{array}\right.
$$


Here

$$
\gamma=\left(\frac{(1-q)^{2}}{2(1+q)}\right)^{\frac{1}{1-q}}
$$

and

$$
y=\varphi_{ \pm}(x)= \pm \int_{0}^{x} \frac{2 \nu(t) d t}{\sqrt{1-\nu^{2}(t)}}, \infty<x<+\infty,
$$

stands for the corresponding free boundary parametrization.

Proof. Let $A(z)$ be a matrix function generated by the complex Beltrami coefficient $\mu$, satisfying (6.1). Since $\mu$ depends on $x$ only, then formulae (5.36), (5.37) represent a unique quasiconformal mapping

$$
\omega(z)=x+i\left(y \pm \int_{0}^{x} \frac{2 \nu(t)}{\sqrt{1-\nu^{2}(t)}} d t\right)
$$

of the complex plane $\mathbb{C}$ onto itself with complex dilatation $\mu$ normalize by $\omega(0)=0, \omega(i)=i, \omega(\infty)=\infty$.

Since $J_{\omega}(z) \equiv 1$, one can apply Corollary 4.2 to represent solutions to the equation (6.3) in the form

$$
u(z)=T(\omega(z))
$$

where $T(w)$ satisfies the equation

$$
\triangle T(w)=T^{q}(w), w=\xi+i \eta .
$$

We see that the function

$$
T(w)=\gamma \eta^{\frac{2}{1-q}}, \quad \text { if } \quad \eta>0
$$

and $T(w)=0$, if $\eta \leq 0$, satisfy the above equation. Thus we arrive at the required solution to the equation (6.3):

$$
u(x, y)=\gamma\left(y \pm \int_{0}^{x} \frac{2 \nu(t)}{\sqrt{1-\nu^{2}(t)}} d t\right)^{\frac{2}{1-q}}, \text { if } y>\varphi_{ \pm}(x), x \in \mathbb{R}
$$

and $u(z)=0$, if $x \leq \varphi_{ \pm}(x)$ where

$$
\gamma=\left(\frac{(1-q)^{2}}{2(1+q)}\right)^{\frac{1}{1-q}}, \quad \varphi_{ \pm}(x)= \pm \int_{0}^{x} \frac{2 \nu(t) d t}{\sqrt{1-\nu^{2}(t)}} .
$$


Let us verify this solution by straightforward computation. Since

$$
u(x, y)=\gamma\left(y-\int_{0}^{x} a_{12}(t) d t\right)^{\frac{2}{1-q}}
$$

we see that

$$
u_{x}=-a_{12} \gamma \frac{2}{1-q}\left(y-\varphi_{+}(x)\right)^{\frac{1+q}{1-q}}, \text { and } u_{y}=\gamma \frac{2}{1-q}\left(y-\varphi_{+}(x)\right)^{\frac{1+q}{1-q}} .
$$

It yields that

$a_{11} u_{x}+a_{12} u_{y}=-a_{12} \gamma \frac{2}{1-q}\left(y-\varphi_{+}(x)\right)^{\frac{1+q}{1-q}}+a_{12} \gamma \frac{2}{1-q}\left(y-\varphi_{+}(x)\right)^{\frac{1+q}{1-q}}=0$.

On the other hand, since $a_{22}-a_{12}^{2}=1$, we get

$$
a_{12} u_{x}+a_{22} u_{y}=\gamma \frac{2}{1-q}\left(y-\varphi_{+}(x)\right)^{\frac{1+q}{1-q}} .
$$

Thus,

$$
\operatorname{div}(A(z) \nabla u)=\left(a_{12} u_{x}+a_{22} u_{y}\right)_{y}^{\prime}=\gamma^{q}\left(y-\varphi_{+}(x)\right)^{\frac{2 q}{1-q}}=u^{q},
$$

and we complete the verification.

\section{Heat equation}

Factorization theorems, similar to Theorem 4.1 and its corollaries, also hold for parabolic and hyperbolic linear and semi-linear equations in the plane, which contain the corresponding divergence operator in the linear part. We give, without proof, one of such results.

Theorem 7.1. Let $\Omega$ be a domain in $\mathbb{C}, A \in M^{2 \times 2}(\Omega)$ and let $f: \mathbb{R} \rightarrow \mathbb{R}$ be a continuous function. Then every weak solution of the semi-linear equation

$$
u_{t}-a^{2} \operatorname{div}[A(z) \nabla u]=f(u) \quad \text { in } \Omega, t>0,
$$

can be represented as the composition

$$
u(z, t)=T(\omega(z), t),
$$

where $\omega: \Omega \rightarrow G$ is a quasiconformal mapping agreed with $A$, and $T$ is a weak solution of the equation

$$
T_{t}-a^{2} J^{-1} \triangle T=f(T) \quad \text { in } G, t>0 .
$$

Here $J$ stands for the Jacobian of the mapping $\omega$. 
As an example for the illustration of Theorem 6.1. we give a statement concerning the heat conductivity equation in divergent form.

Proposition 7.1. Let $\mathbb{D}$ be the unit disk and let the matrix function $A(z)$ be generated by the Beltrami coefficient

$$
\mu(z)=k(|z|) \frac{z}{\bar{z}}
$$

in accordance with formula (2.8) where

$$
k(t)=\nu^{2}(t) \pm i \nu(t) \sqrt{1-\nu^{2}(t)}
$$

and $\nu(t), 0 \leq t<1$, stands for an arbitrary measurable real-valued function, such that $|\nu(t)| \leq q<1$. Then the fundamental solution to the inhomogeneous anisotropic heat equation

$$
u_{t}-a^{2} \operatorname{div}(A(z) \nabla u)=0, \quad z \in \mathbb{D}, t>0
$$

has the representation

$$
u(z, t)=\frac{1}{4 \pi a^{2} t} \exp \left(-\frac{|z|^{2}}{4 a^{2} t}\right), z \in \mathbb{D}, t>0 .
$$

Proof. We are looking for a solution in the form $u(z, t)=T(\omega(z), t)$, where $\omega(z)$ stands for a quasiconformal automorphism of the unit disk $\mathbb{D}$ agreed with the matrix function $A(z)$. Since the mapping $w=\omega(z)$ is volume preserving, we see that its Jacobian is identically equal to the unit. Then, by Theorem $7.1, T(w, t)$ will satisfy the equation

$$
T_{t}-a^{2} \triangle T=0, w \in \mathbb{D}, t>0
$$

It is a classical result that the fundamental solution to the inhomogeneous heat equation (7.8) has the representation

$$
T(w, t)=\frac{1}{(2 a \sqrt{\pi t})^{2}} \exp \left(-\frac{|w|^{2}}{4 a^{2} t}\right), w \in \mathbb{D}, t>0
$$

Since in our case $|\omega(z)|=|z|$, we arrive at the required conclusion. 
Remark 7.1. In a similar way we can study the well-known semi-linear combustion equation in an anisotropic medium

$$
u_{t}-a^{2} \operatorname{div}[A(z) \nabla u]=e^{u}
$$

by means of reducing its weak solution to the composition of a weak solution in the isotropic case, see e.g. [4] and [30] and the references therein,

$$
T_{t}-a^{2} J^{-1} \triangle T=e^{T}
$$

with a quasiconformal mapping $\omega$ generated by $A$. As above, $J$ stands for the Jacobian of the mapping $\omega$.

\section{Boundary value problems}

The Factorization Theorem 4.1 allows us, in particular, to reduce the study of the boundary value problems, say the Dirichlet problem

$$
\left\{\begin{array}{cl}
\operatorname{div}(A(z) \nabla u)=f(u) & \text { if } z \in \Omega, \\
u=\varphi, & \text { if } z \in \partial \Omega
\end{array}\right.
$$

in a Jordan domain $\Omega$ with $A \in M^{2 \times 2}(\Omega)$ and continuous boundary function $\varphi$ to the study of the following Dirichlet problem in the unit disk

$$
\left\{\begin{array}{cc}
\triangle T=J(w) f(T) & \text { if }|w|<1, \\
T=\psi, & \text { if }|w|=1
\end{array}\right.
$$

with the corresponding boundary function $\psi=\varphi \circ \omega^{-1}$ where $\omega$ is a quasiconformal mapping of $\Omega$ onto the unit disk $\mathbb{D}$ agreed with $A$, and $J$ is the Jacobian of $\omega^{-1}$. First of all, note that $\omega$ exists by the Measurable Riemann mapping theorem, see e.g. Theorem V.B.3 in [1] and Theorem V.1.3 in [25].

The existence and continuity of the boundary function $\psi$ in the case of an arbitrary Jordan domain $\Omega$ is a fundamental result of the theory of the boundary behavior of conformal and quasiconformal mappings. Namely,

$$
\omega^{-1}=h \circ g
$$

where $g$ stands for a quasiconformal automorphism of the unit disk $\mathbb{D}$ and $h$ is a conformal mapping of $\mathbb{D}$ onto $\Omega$. It is known that $g$ can be extended to a homeomorphism of $\overline{\mathbb{D}}$ onto itself, see e.g. Theorem I.8.2 in [25]. Moreover, by the well-known Caratheodory-Osgood-Taylor theorem on the boundary correspondence under conformal mappings, see [8] and [28], the mapping $h$ is extended to a homeomorphism of $\overline{\mathbb{D}}$ onto $\bar{\Omega}$. Thus, the function $\psi$ is well defined and really continuous on the unit circle. 


\section{References}

[1] L. V. Ahlfors, Lectures on quasiconformal mappings, Princeton, N.J., Van Nostrand, 1966; Reprinted by Wadsworth Ink. Belmont, 1987.

[2] K. Astala, T. Iwaniec, G. Martin, Elliptic partial differential equations and quasiconformal mappings in the plane, Princeton Mathematical Series, 48, Princeton University Press, Princeton, NJ, 2009.

[3] C. Bandle, M. Marcus, Asymptotic behavior of sulutions and their derivative for semilinear elliptic problems with blow-up on the boundary // Ann. Inst. H. Poincare Analyse Non Lineaire, 12 (1995), No. 2, 155-171.

[4] G. I. Barenblatt, Ja. B. Zel'dovic, V. B. Librovich, G. M. Mahviladze, Matematicheskaya teoriya goreniya i vzryva, Nauka, Moscow, 1980; transl. in "The mathematical theory of combustion and explosions", Consultants Bureau, New York, 1985.

[5] L. Bieberbach, $\triangle u=e^{u}$ und die automorphen Funktionen // Math. Ann., 7 (1916), 173-212.

[6] B. V. Bojarski, Generalized solutions of a system of differential equations of the first order of the elliptic type with discontinuous coefficients, Mat. Sb. 43 (85) (1957), 451-503.

[7] B. Bojarski, V. Gutlyanskii, O. Martio, V. Ryazanov, Infinitesimal geometry of quasiconformal and bi-lipschitz mappings in the plane, EMS Tracts in Mathematics, 19. European Mathematical Society (EMS), Zurich, 2013.

[8] C. Caratheodory, Uber die gegenseitige Beziehung der Rander bei der konformen Abbildungen des Inneren einer Jordanschen Kurve auf einen Kreis // Math. Ann., 73 (1913), 305-320.

[9] J. I. Diaz, Nonlinear partial differential equations and free boundaries, Vol. I, Elliptic equations, Research Notes in Mathematics, 106, 1985.

[10] V. Gol'dshtein, L. Gurov, A. Romanov, Homeomorphisms that induce monomorphisms of Sobolev spaces // Israel J. Math., 91 (1995), No. 1-3, 31-60.

[11] V. Gol'dshtein, A. Ukhlov, About homeomorphisms that induce composition operators on Sobolev spaces // Complex Var. Elliptic Equ. 55 (2010), No. 8-10, $833-845$.

[12] G. M. Goluzin, Geometric theory of functions of a complex variable, Amer. Math. Soc., Providence, Rhode Island, 1969.

[13] V. Ya. Gutlyanskiı, O. Martio, Conformality of a quasiconformal mapping at a point // J. Anal. Math. 91 (2003), 179-192.

[14] V. Gutlyanskiı̌, O. Martio, Rotation estimates and spirals // Conform. Geom. Dyn., 5 (2001), 6-20.

[15] V. Gutlyanskii, O. Nesmelova, V. Ryazanov, Semilinear equations in the plane and quasiconformal mappings // Dopov. Nats. Akad. Nauk Ukr. Mat. Prirodozn. Tekh. Nauki, (2017), No. 1, 8-16.

[16] V. Gutlyanskii, O. Nesmelova, V. Ryazanov, On a model semilinear elliptic equation in the plane // Ukr. Mat. Visn., 13 (2016), No. 1, 91-105; transl. in J. Math. Sci. (US) 220 (2017), No. 5, 603-614.

[17] V. Ya. Gutlyanskii, V. I. Ryazanov, On the theory of the local behavior of quasiconformal mappings // Izv. Ross. Akad. Nauk Ser. Mat., 59 (1995), No. 3, 31-58; transl. in Izv. Math. 59 (1995), No. 3, 471-498. 
[18] V. Ya. Gutlyanskii, V. I. Ryazanov, The geometric and topological theory of functions and mappings, Kiev, Naukova Dumka, 2011.

[19] V. Gutlyanskii, V. Ryazanov, U. Srebro, E. Yakubov, The Beltrami Equation: A Geometric Approach, Developments in Mathematics, 26, Springer, New York, 2012.

[20] J. B. Keller, On solutions of $\triangle u=f(u) / /$ Comm. Pure Appl. Math., 10 (1957), 503-510.

[21] V. A. Kondratiev, E. M. Landis, Semilinear equations of the second order with non-negative characteristic form // Mat. Zam., 44 (1988), No. 4, 457-468.

[22] A. A. Kovalevskii, I. I. Skrypnik, A. E. Shishkov, Singular solutions of nonlinear elliptic and parabolic equations, De Gruyter Series in Nonlinear Analysis and Applications, 24, 2016.

[23] M. Marcus, L. Veron, Nonlinear second oder elliptic equations involving measures, De Gruyter, Series in nonlinear analysis and applications, 21, Berlin-Boston, 2014.

[24] O. A. Ladyzhenskaya, N. N. Uraltseva, Linear and quasilinear elliptic equations, Nauka, Moscow, 1964.

[25] O. Lehto, K. I. Virtanen, Quasiconformal mappings in the plane, 2-nd Edition, Springer-Verlag, Berlin-Heidelberg-New York, 1973.

[26] A. V. Neklyudov, Behavior of solutions to Gauss-Bieberbach-Rademacher equation on plane // Ufa Math. J., 6 (2014), No. 3, 85-94.

[27] O. A. Oleinik, The equation $\triangle u+k(x) e^{u}=0 / /$ Uspehi Mat. Nauk, 33 (1978), No. 2 (200), 203-204.

[28] W. Osgood, E. Taylor, Conformal transformations on the boundaries of their regions of definition // Trans. Amer. Math. Soc. 14 (1913), 277-298.

[29] R. Osserman, On the inequality $\triangle u \geq f(u)$ // Pacific J. Math., 7 (1957), 16411647.

[30] S. I. Pokhozhaev, On an equation of combustion theory // Mat. Zametki, 88 (2010), No. 1, 53-62 ; transl. in Math. Notes, 88 (2010), No. 1-2, 48--56.

[31] H. M. Reimann, T. Rychener, Funktionen beschränkter mittlerer Oszillation, Lecture Notes in Mathematics, 487, Springer-Verlag, Berlin-New York, 1975.

[32] Yu. G. Reshetnyak, Prostranstvennye otobrazheniya s ogranichennym iskazheniem, Nauka, Novosibirsk, 1982; Space mappings with bounded distortion, Transl. Math. Mon., 73. AMS, Providence, RI, 1989.

[33] S. L. Sobolev, On some transformation groups of an n-dimensional space, Dokl. Akad. Nauk SSSR, 32 (1941), No. 6, 380-382.

[34] A. Ukhlov, Mappings that generate embeddings of Sobolev spaces // Sibirsk. Mat. Zh., 34 (1993), No. 1, 185-192; transl. in Siberian Math. J., 34 (1993), No. 1, $165-171$.

[35] I. N. Vekua, Some properties of solutions of Gauss equation // Trudy Mat. Inst. Steklov, 64 (1961), 5-8.

[36] S. K. Vodop'yanov, On the regularity of mappings inverse to the Sobolev mapping // Mat. Sb. 203 (2012), No. 10, 3-32; transl. in Sb. Math. 203 (2012), No. 9-10, 1383-1410. 
[37] S. K. Vodop'yanov, N. A. Evseev, Isomorphisms of Sobolev spaces on Carnot groups and quasi-isometric mappings // Sibirsk. Mat. Zh., 55 (2014), No. 5, 1001-1039; transl. in Sib. Math. J., 55 (2014), No. 5, 817-848.

[38] S. K. Vodop'janov, V. M. Gol'dshtein, Lattice isomorphisms of the spaces $W_{n}^{1}$ and quasiconformal mappings // Sibirsk. Mat. Zh., 16 (1975), 224-246.

[39] S. K. Vodopyanov, A. Ukhlov, Sobolev spaces and $(P, Q)$-quasiconformal mappings of Carnot groups // Sib. Mat. Zh., 39 (1998), No. 4, 665-682; transl. in Siberian Math. J., 39 (1998), No. 4, 665-682.

\section{Contact information}

Vladimir

Gutlyanskir,

Olga Nesmelova,

Vladimir Ryazanov
Institute of Applied Mathematics

and Mechanics of the NAS of Ukraine,

Slavyansk, Ukraine

E-Mail: vgutlyanskii@gmail.com,

star-o@ukr.net,

vl.ryazanov1@gmail.com 